\title{
MULTIPLE MODEL BASED FLIGHT CONTROL DESIGN
}

\author{
Geetha K. Thampi ${ }^{1}$, Jose C. Principe ${ }^{1}$, Mark A. Motter ${ }^{2}$, JeongHo Cho ${ }^{1}$, Jing Lan ${ }^{1}$ \\ ${ }^{1}$ Computational NeuroEngineering Laboratory, University of Florida, Gainesville, FL 32611 \\ ${ }^{2}$ NASA Langley Research Center, Hampton, Virginia, 23681-2199
}

\begin{abstract}
Flight control system for high performance aircraft has been an area of intense research. There is a need for sophisticated control strategies that aim for better performance under severe flying conditions. The coupled and time varying dynamics of these systems make the identification problem complicated. This paper addresses the problem of modeling and controlling non-linear systems by utilizing a selforganizing map (SOM). The proposed method uses multiple models of the non-linear plant for identification, as well as a multi-model controller. The mathematical formulation of the controller algorithm and the switching strategy are discussed. Preliminary results of using the proposed strategy for the identification and control of a high performance aircraft are presented to demonstrate the versatility of the algorithm.
\end{abstract}

\section{INTRODUCTION}

Many systems especially aircrafts have dynamics, which vary considerably over the operating regime for a single controller to meet the design specifications. Aircraft Flight Control design has been dominated by classical control techniques. Traditional flight control designs involve linearizing the vehicle dynamics about several operating conditions throughout the flight envelope, designing linear controllers for each condition and blending these point designs with an interpolation scheme [1]. While this tradition, though rather tedious, has produced many highly reliable and effective control systems in the past, recent years have seen a growing interest in the use of robust, non-linear adaptive control theory for flight control [2]. There are a number of flight control applications reported in the literature, which exploit the on-line learning ability of neural networks [3,4,5]. Most of the adaptive methods explicitly account for the intrinsic non-linearities in the system. This explains the growing interest in the development of real time adaptive methods for flight control problems where the aircraft characteristics are poorly understood, and are highly nonlinear. The work presented here investigates the possibility of using multiple adaptive controllers for the LOFLYTE aircraft, which has nonlinear and time varying dynamics. Multiple models are used to identify the system over the whole flight envelope and the models are switched according to the change in the dynamics. The objective is to design a group of controllers corresponding to each of these models, which will stabilize and compensate for the entire system by adaptive control laws through the optimization of a cost function, which is the square of the deviation error between actual and desired output of the controlled plant.

The concept of multiple models and switching between the models has been an area of interest in Control Theory. Multiple Kalman filter-based models [6] were studied in the past to improve the accuracy in state estimation and control problems. In recent years multiple models using neural networks have been used with switching between the models $[7,8,9]$. Principe et al $[10,11]$ have successfully modeled a chaotic time series using multiple models and applied it to the set point regulation of a NASA Langley wind tunnel during the aerodynamic testing of model aircraft. Inspired by this approach, a new train of thought based on the selforganizing map (SOM) for system identification has emerged. This method identifies the plant at different segments of the state space trajectory, and associates a model for each of them, giving rise to the concept of multiple models structured by an SOM. Here the global dynamics is approximated by a preset number of local linear models, which are concurrently derived through competition using Kohonen's SOM. This in turn is used as the building block for the design of the controller. So instead of one neurocontroller as in other adaptive schemes, we will have a group of controllers associated with each identified model, thus taking care of the system over the whole operating regime. The controllers are designed independently of the others in contrast to the gain scheduling method, which is an interpolated scheme. A preliminary study of the use of multiple models for some simple nonlinear systems is done in [12].

\section{ADAPTIVE INVERSE CONTROL IN A MULTIPLE MODEL ENVIRONMENT}

Tracking based on inverse control is a continuing area of research in control theory [13]. The basic objective of adaptive inverse control is to determine the control input such that the system output follows a specified trajectory. An error signal, the difference between the plant output and the command input is used by an adaptive algorithm to adjust the controller's parameters to minimize the mean square of 
this error. Sometimes it is desired that the plant output track not the command input but a delayed or smoothed version of the command input. This is called the model reference adaptive inverse control system. The general block diagram of a model reference adaptive inverse control is shown in figure 1. The reference model can be chosen to have the same dynamics that the designer would like to have for the entire system. For the flight control problem, the reference model is chosen in such a way that the poles of the model be in accordance with the handling specification.

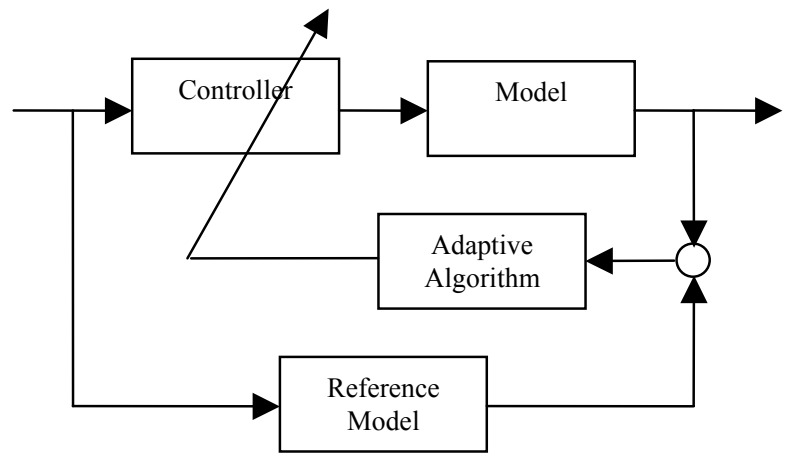

Figure 1. Model Reference Adaptive Inverse Control

After convergence the cascade of the controller and the plant would have a dynamic response as that of the reference model. The first step in the design of any control system is the identification of the system. As was told before this is done using SOM. The input-output data from the simulator is fed to the SOM. The clustering property of the SOM is combined with least square method to produce a group of models each of which locally represents the dynamics of this nonlinear system. The development of local models based on SOM is elaborated in detail in [14] and so will not be addressed here. Since we identify the plant using multiple models, it is necessary to associate these models with a corresponding controller. In a multiple model context the model shown in figure 1 will be one of the models identified and it is desired to design the corresponding controller. The block diagram for a single controller design is shown in figure 2. As stated before our principal objective is to determine a control input $u(k)$, which will result in the output $y(k)$ of the plant in (1) tracking a specified sequence $y^{*}(k)$ with sufficient accuracy. The system has $N$ identification models denoted by $\left\{M_{r}\right\}_{r=1}^{N}$, in parallel. Corresponding to each model $M_{r}$, a controller $C_{r}$ is designed such that $C_{r}$ achieves the control objective for $M_{r}$. So at every instant one of the models is selected by the SOM and the corresponding controller is used to control the actual system. This is the switching part of the system. The controller is trained using gradient descent learning. This means that the output plant error must be propagated through the multiple models to adapt the parameters of the controller. Since the multiple models are structured by the SOM, we have to derive the dual of the SOM for sensitivity propagation. Since the SOM input/output map is discontinuous, it may appear that it is impossible to obtain its dual system. Albeit strictly speaking this is true, practically it is possible to transfer sensitivities through a SOM, provided sample-by-sample estimates are used like in the LMS algorithm. In the LMS we will be propagating instantaneous errors and so the algorithm will not see the discontinuities. To design $C_{r}$, the linear model $M_{r}$ is taken and the controller is designed adaptively using LMS algorithm [15]. The block diagram associated with the design of the controller is shown in the figure 2 .

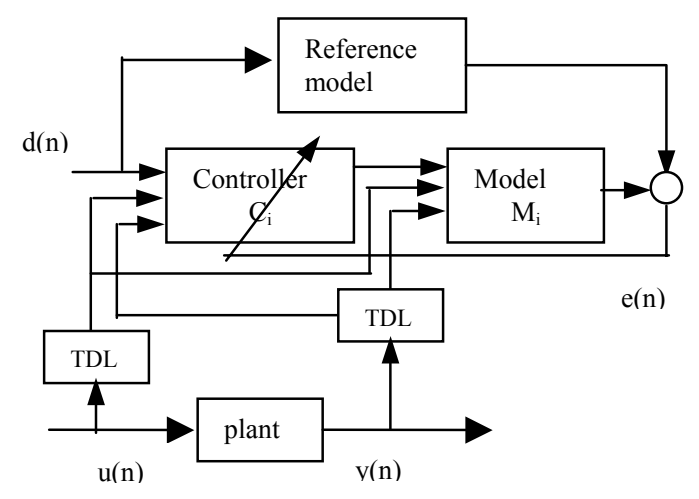

Figure 2. Single controller design

Let the desired output be $d(n)$ and the controller weight vector $\mathbf{w}_{\mathbf{c}}(n)$. Then the controller output $u(n)$ is given by,

$u(n)=\mathbf{w}_{c}^{T}(n) \overline{\mathbf{d}}(n)$,

where $\overline{\mathbf{d}}(n)=[d(n) y(n-1)]^{T}$, the overall controller input.

The corresponding SOM output is the winner PE weight, $w^{*}(n)$, which is applied to the model $M_{i}$ (with parameter vector $\left.\mathbf{a}_{i}\right)$ to get a response $\hat{y}(n+1)$. The controller weight vector is adapted using simple LMS rule, which utilizes the instantaneous gradient. The instantaneous gradient is defined as

$J(n)=\frac{1}{2} e^{2}(n)$

So the weight vector update equation is

$\mathbf{w}_{\mathbf{c}}(n+1)=\mathbf{w}_{\mathbf{c}}(n)-\eta \frac{\partial J(n)}{\partial \mathbf{w}_{\mathbf{c}}(n)}$

We can compute this gradient by using chain rule. 


$$
\begin{aligned}
\frac{\partial J(n)}{\partial \mathbf{w}_{\mathbf{c}}(n)} & =e(n) \frac{\partial e(n)}{\partial \mathbf{w}_{\mathbf{c}}(n)} \\
& =-e(n) \frac{\partial \hat{y}(n)}{\partial \mathbf{w}_{\mathbf{c}}(n)} \\
& =-e(n) \mathbf{a}_{i} \frac{\partial \mathbf{u}(n)}{\partial \mathbf{w}_{\mathbf{c}}(n)} \\
& =-e(n) \mathbf{a}_{i} \overline{\mathbf{d}}(n)
\end{aligned}
$$

The adaptation process is repeated for all the models resulting in a model controller pair $\left\{M_{i}, C_{i}\right\}$ associated with

every SOM PE. As mentioned before the natural way to decide when and to which controller one should switch is to determine the model that best describes the plant. This will be decided by the SOM in accordance with the present state of the system. In a nutshell, the procedure for controlling the non-linear plant is as follows: SOM determines the present model of the system corresponding to the present state variables and control inputs and this in turn will fire the corresponding controller. The controller output is then fed to the actual system, which is forced to track the reference trajectory.

In order to accentuate the superiority of using multiple models and controllers to identify the dynamics and controlling a nonlinear system, another experiment is carried out replacing the SOM with a TDNN for the nonlinear system identification. The TDNN employed in this work has one hidden layer with 5 neurons and tanh nonlinearity and one linear output neuron. It has 6 inputs; two of them correspond to the control inputs and the other four to the delayed values of the desired response. The network is trained for 100 epochs using 5400 samples of data with an adaptive step size. The identified TDNN is used for the indirect learning of the model reference controller. The structure of the model-based neuro-controller is shown in figure 3.

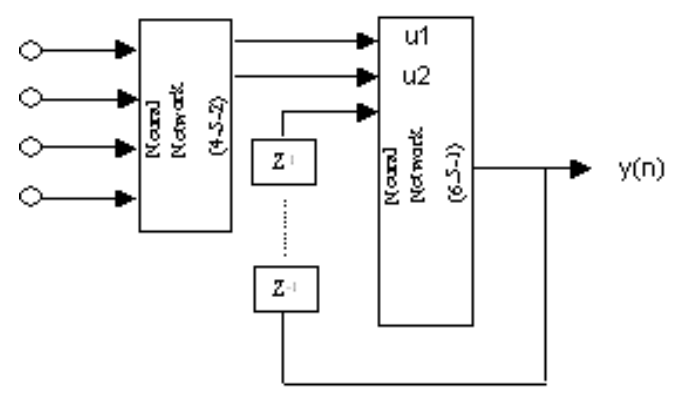

Figure 3. NeuroController architecture

The controller structure is chosen to be 4-5-2, with tanh nonlinearity at the hidden and output layer. The delayed samples of the command input are the inputs to the controller. The controller is trained for 100 epochs using
5000 data samples using back propagation through the identified TDNN model.

\section{SIMULATION RESULTS}

The LOFLYTE aircraft model designed by Accurate Automation Corporation (AAC) is used to verify the effectiveness of the proposed approach. The input-output data is used to model the dynamics of the system through SOM based local modeling. This paper uses the models estimated by a companion paper presented in this conference [14]. The design of the controllers is carried out for each of these local models as discussed in the previous section. The simulations results are shown in figure 4 .

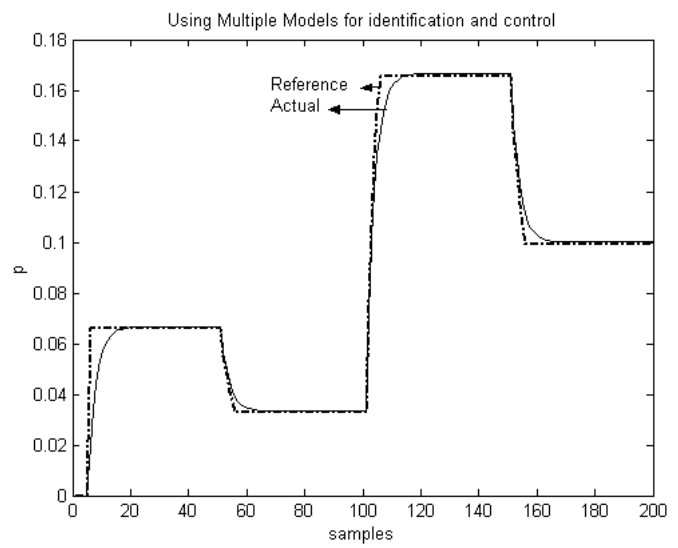

Figure 4. Tracking of pitch rate for a command

Each of the controllers are simple in structure, to be precise an FIR of order 4. As can be seen from the figure the plant output exactly follows the desired output. The result of using the conventional neurocontroller is shown in figure 5.

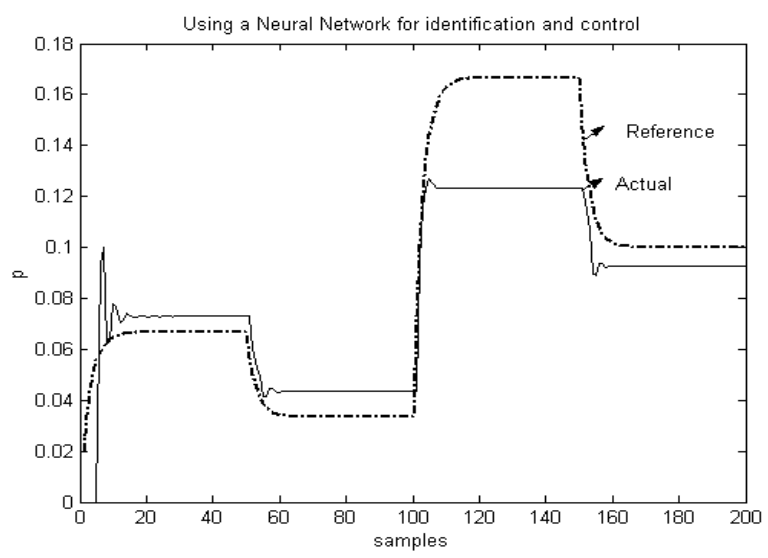

Figure 5 Tracking of pitch rate for a command

As can be seen a single neurocontroller is unable to control the system with a high degree of accuracy, though it is able to track the changes. On the other hand the multiple model- 
based controllers are doing a good job in tracking the desired signal, since they know the precise dynamics at each point and are able to compensate for them with the corresponding controllers in contrast to the neuro-controllers that learn the global dynamics of the entire system. Thus, the neurocontrollers lose the information regarding the local dynamics.

\section{SUMMARY}

We have shown results of the use of SOM based multiple controllers in a flight control context. Our preliminary work indicates that the SOM can identify the highly nonlinear aircraft dynamics and assist the controller in inverting the dynamics so that the overall system with the controller tracks the desired signal perfectly well. The adaptation of the controller can be done offline. The overall system seems to be stable although there is no rigorous proof for the overall stability. In this paper we used a switching strategy depending on the values of the inputs to the SOM or the present state of the system. We can also evaluate a performance index and switch the models according to this index. One disadvantage of using SOM is the inherent limitation of any memory-based method. Hence, on the flop side, SOM needs a fairly good amount of data for training.

The comparison of the proposed method with a single neurocontroller exemplifies the power and simplicity of our approach. The multiple controllers excel in their simple structure, accuracy and response speed.

It has been demonstrated in the literature that the Inverse of the Best Estimator (IBE), which is the indirect learning, is superior to the Best Inverse Estimator (BIE) [16]. What we have used is exactly the IBE since we model the dynamics of the system and propagate the error through the model for the controller training.

Acknowledgements: This work was partially supported by Accurate Automation Corporation under grant \#463 and NASA grant NAG-1-02068.

\section{REFERENCES}

[1] Lee. C. H., Chung. M. J., "Gain Scheduled State Feedback Control Design Technique for Flight Vehicles", IEEE Transactions on Aerospace and Electronic Systems, Vol. 37, No. 1, January 2001, pp. 173-182.

[2] B. S. Kim, Anthony. J. Calise, "Nonlinear Flight control Using Neural Networks", Journal of Guidance, Control and Dynamics, Vol. 20, No. 1, January-February 1997.

[3] Troudet. T, Garg. S, Merril. W., "Neural Network Application to Aircraft Control System Design," Journal of Guidance, Control, and Dynamics, Vol. 16, No. 4, 1993, pp. 738-747.
[4] Sadhukhan. D., Fateih S., "F8 Neurocontroller Based on Dynamic Inversion", Journal of Guidance, Control and Dynamics, Vol. 19, No. 1, 1996, pp. 150-156.

[5] Napolitano M. R., Kincheloe M, "On-Line Learning Neural Network Controllers for Autopilot Systems," Journal of Guidance, Control and Dynamics, Vol. 18, No. 6, 1995, pp. 1008-1015.

[6] D. G. Lainiotis, (1976) "Partitioning: A Unifying Framework for Adaptive Systems", Proceedings of the IEEE, 64:11261143,1182-1197, August 1976.

[7] K. S. Narendra, S. Mukhopadhyay, (1997) "Adaptive Control using Neural Networks and Approximate Models", IEEE Transactions on Neural Networks, 475-485, Vol. 8, No. 3, May 1997.

[8] K. S. Narendra, J. Balakrishnan, (1997) "Adaptive Control using Multiple Models", IEEE Transactions on Automatic Control, 171-187, Vol. 42, No. 2, February 1997.

[9] K. S. Narendra, J. Balakrishnan, M. K. Ciliz, (1995)“Adaptive and Learning using Multiple Models, Switching and Tuning”, IEEE Control Systems, 37-50, June 1995.

[10] J. C. Principe, L. Wang, M. A. Motter, (1998)“Local Dynamic Modeling with Self-Organizing Maps and Applications to Nonlinear System Identification and Control", Proceedings of IEEE, 2240-2258 Vol. 86, No. 11, November 1998.

[11] M. Motter (1997), "Control of the NASA transonic wind tunnel the self-organizing feature map," Ph.D. dissertation, Univ. of Florida, Gainesville, Dec. 1997

[12] Thampi G. K., Principe J. C., Cho J., Erdogmus D., Motter M. A., "Adaptive Inverse Control Using SOM based Multiple Models", to appear in Controlo 2002

[13] B. Widrow, E. Walach, (1996), "Adaptive Inverse Control," Prentice Hall Information \& System Science Series, NJ

[14] Cho. J., Lan. J., Thampi G., Principe J. C., Motter M. A,'Identification of Aircraft Dynamics Using a SOM and Local Linear Models", to be published in MWSCAS Aug 2002.

[15] B. Widrow, S. D. Stearns (1985), “ Adaptive Signal Processing, Prentice-Hall, Englewood Cliffs, NJ.

[16] Karniel. A., Meir. R., Inbar. G. F., "Best estimated inverse versus the inverse of the best estimator", Neural Networks Vol. 14, 2001, pp. 1153-1159 